\title{
Electrical Resistivity Tomography for Magnetic Flowmeter
}

\author{
Hencken, Kai \\ Schrag, Daniel \\ ABB Switzerland Ltd, \\ Baden Dättwil (Switzerland) \\ Grothey, Harald \\ ABB Automation Products $\mathrm{GmbH}$ \\ Göttingen (Germany)
}

\section{Introduction}

Magnetic flowmeter are widely used in the automation industry to measure the volume flow of conductive liquids, especially of water. They are robust devices with no moving part and with almost no obstable hindering the flow. The basic principle of the flowmeter is the magnetic induction. The flow of the conductive medium through a magnetic field orthogonal to the flow induces an electric field. The resulting voltage difference is measured with two electrodes at both sides of the flowmeter tube. This voltage is proportional to the average velocity of the medium, if the magnetic field is homogenous and if the flow profile is axial symmetric [1,2]. An accurate flow measurement is therefore possible if the pipe is fully filled. This full-filling is often controlled with the help of an additional electrode at the top of the tube.

Especially in waste water applications, the full-filling of the pipe cannot be guaranteed, as the flow rates vary widely and the tube diameter is dimensioned towards the maximum flow. One option is then to mount the flowmeter in a way that the full-filling can be achieved also at low flow rates. But this often involves the use of bend tubes or other obstacles in the flow.

Therefore magnetic flowmeter with the ability to measure in partially filled pipes are of interest. Magnetic flowmeters are a good option as long as two requirements are met: First the pair of measurement electrodes needs to be in contact with the liquid also for low filling heights. The position of the lowest pair of electrodes then limits the lowest possible height, which can be used for measurements. Electrodes at higher levels on the other hand are an advantage, as the voltage difference measured by them is larger. Second a system to measure the filling height is required. In principle any level measurement principle can be used here. The flow velocity is then corrected for the effective area and the total volume flow calculated. Different techniques using ultrasonic or capacitive level measurement techniques and inhomogenous magnetic field configurations have been proposed and are in use for this.

\section{The PARTI-MAG Magnetic Flowmeter for Partially Filled Pipes}

The PARTI-MAG flowmeter of ABB is a magnetic flowmeter for partially filled pipes. It uses a multielectrode configuration with 8 (9) electrodes. These electrodes are used for both the flow and for the level measurement, see Fig. 1 [3]. For this a current is injected into a pair of electrodes and voltages on two other electode pairs are measured. The two voltages are sensitive to the filling level. As they are also dependent on the conductivity of the medium, the ratio of the two voltages is taken. A curve translates this voltage ratio into a height and into a correction factor for the flow rate. An advantage of this approach is that the electrode pairs are needed already for the flow measurement and no additional level detection system is needed. A limitation of this measurement system comes from the fact that a reasonable number of electrode needs to be present in order to measure the height. It is an advantage to make voltage measurements on electrode pairs, that do not carry a current, as otherwise the electrode-electrolyte interface will lead to an additional voltage drop, which is not very well controlled and needs to be corrected for.

\section{The Principle of Electrical Resistivity Tomography}

Electrical Resisitivity Tomography (ERT) is a special case of Electrical Impedance Tomography (EIT), which is one of the important examples of "soft-field tomography". Tomography quite generally is a technique to reconstruct the distribution of a property in the interior of a system by analyzing measurements done at the perimeter. The best known example is $x$-ray tomography, where the measurement of the attenuation of $x$-rays in a system is used to determine material distributions inside, e.g., the human body. As the x-rays in this case follow a straight line, this is known as an example of "hard-field tomography". 


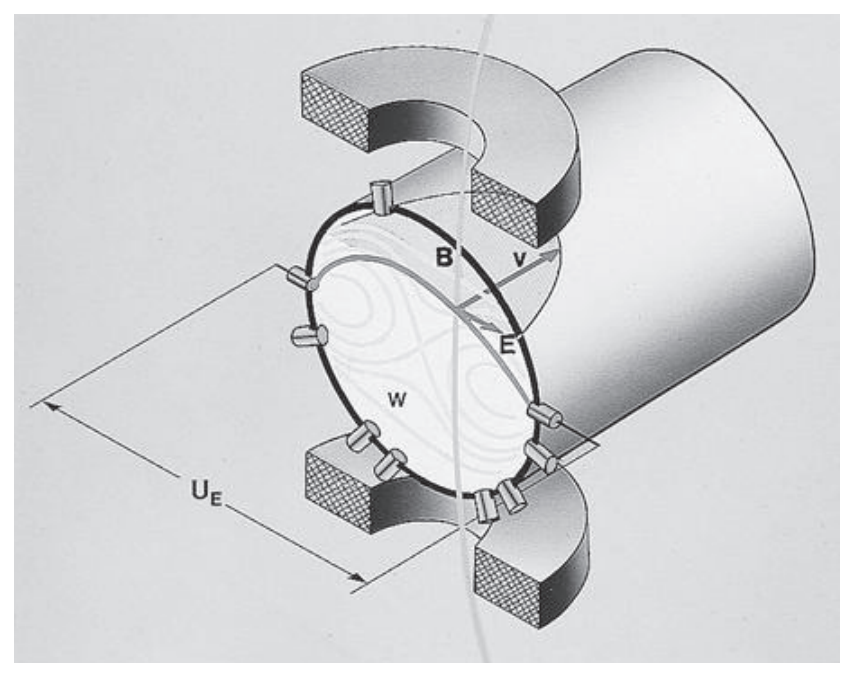

Figure 1: The ABB PARTI-MAG magnetic flowmeter for partially-filled pipes [3].

In the case of ERT (or EIT), one makes a number of measurements of voltages at electrodes at the outside, e.g., of a pipe, when current is injected on some other electrodes. In our case at low frequencies, the impedances are almost real, whereas at high frequency the dielectric properties of the medium come into play. In this way different materials can be distinguished.

This measurement technique has found a number of application as "Industrial and Process Tomography", especially in the area of multi-phase flow, where different materials are distinguished in this way. For details of the large body of applications, we refer to [4].

The reconstruction of the conductivity in ERT is an ill-posed problem. "Ill-posed problems" are problems, which have a bad stability behavior with respect to distortions of the measured results. In addition the problem is also underdetermined, as the conductivity distribution can in principle be arbitrary as a function of the position in the pipe, whereas only a finite (small) number of measurements are made on the outside. Therefore special numerical techniques are needed to reliably reconstruct the conductivity in this way [5].

To solve this reconstruction problem two parts are needed. On the one hand one needs to solve the "forward problem". In our case this is the calculation of the voltages on the electrodes for different current patterns with the conductivity known. For this one solves the continuity equation for the electrical potential $\phi(\vec{x})$ inside the pipe

$$
\operatorname{div}[\sigma(\vec{x}) \operatorname{grad}(\phi(\vec{x}))]=0,
$$

together with appropriate boundary conditions, corresponding to isolating regions of the pipe or to the electrodes. This is typically done by using a FE discretization scheme on a grid. With this the voltages for a given conductivity profile are obtained. In order to solve the inverse problem a non-linear optimization problem is solved. One determines the conductivity pattern, which best reproduces the measured result by solving

$$
\arg \min _{\sigma(x)}\left|\mathbf{U}_{\text {meas }}-\mathbf{U}_{f w d}(\sigma)\right|^{2}
$$

where $\mathbf{U}_{\text {meas }}$ is the vector of measured voltages and $\mathbf{U}_{f w d}(\sigma)$ the voltages as calculated from the forward problem. As mentioned above this is not a well-posed problem, as small errors in the measurements produce large changes in the conductivity distribution found. In addition these are often not realistic.

In order to cope with this the conductivity distribution is regularized. For this the expected shape of the conductivity distribution is taken into account by using so-called "priors". These priors capture the characteristics of the expected conductivity distributions. They are added to the reconstruction algorithm in the following way:

$$
\arg \min _{\sigma(x)}\left[\left|U_{\text {meas }}-U_{f w d}(\sigma)\right|^{2}+\lambda M(\sigma)\right]
$$

The parameter $\lambda$ determines the strength of the regularization and $M(\sigma)$ is the prior to be used. 
With EIDORS [6,7] there exists an open-source system for the reconstruction, suited for the use in this kind of problems. Eidors has been developed over a number of years. It is seen as a reference implementation for the algorithms used in this field. Due to the availability of the source extensions to the system are easily possible. We have therefore made use of this system here.

\section{Application of ERT to Level Measurement in Partial Filled Pipes}

We study here the applicability of tomographic reconstruction principles to the determination of the filling height of a partial filled magnetic flowmeter. The multi-electrode system of the PARTI-MAG is already close to the typically found tomographic systems used in this field. It has two main limitations: The electrodes are distributed only in the lower half of the pipe, and the area of the electrodes is rather small compared to the diameter of the pipe. Therefore we see our investigation as a study how well the system is suitable for such an application. Our main focus is the determination of the conductivity distribution with respect to a determination of the phase transition between liquid and air, even though other possibilities can be studies as well.

As air is non-conductive, we can only make use of those electrodes, which are connected to the liquid. Therefore we restrict ourself here to filling levels above $50 \%$. We also note that in our system the electrodes are distributed in one plane only. Therefore we will not be able to resolve any conductivity pattern along the pipe. This is in contrast to typical tomographs, which often have several layers of electrodes, see e.g. [4]. Therefore we have restricted ourself mainly to 2D reconstruction techniques, that is, assuming, that the problem can be described by a $2 \mathrm{D}$ system. This is not an intrinsic limitation, see below for the possibility to study it as a 3D problems.

For the measurements we have used electrode configurations which inject currents and measure voltages mostly on opposite sides, as we expect these to be most sensitive to the conductivity distribution throughout the pipe.

As mentioned above it is essential for the reconstruction to use appropriate prior distributions in order to be able to get meaningful results. EIDORS already provides some of the prior distributions typically used. One of them is, for example, the Tikhonov prior, which is the simplest one and often used, if one searches for small variations in the conductivity. It is given by

$$
M_{\text {Tikhonov }}(\sigma)=\sum_{i}\left(\sigma\left(x_{i}\right)\right)^{2}=\sum_{i} \sigma_{i}^{2},
$$

Where the $x_{i}$ are the center positions of our discretization grid. This prior favors small deviations of the conductivity from a reference value. Such a prior doesn't seem to be very realistic in our case. Even though we have homogenous conductivities in both phases (liquid and air), they differ substantially. Therefore we don't expect this prior to give us reasonable results. The result of the reconstruction of a conductivity pattern with this prior can be seen in Fig. 2(a). As expected the reconstructed results are not similar to the real one. The regularization prefers conductive regions close to the electrodes, but small conductivities away from them. Still on the average a higher conductivity is fond in the lower region compared to the higher one.

Another prior, that is often used, is the Laplace prior. It regularizes the conductivity distribution to be smooth. Differences of the conductivity in neighboring cells are suppressed. For this the prior is given by

$$
M_{\text {Laplace }}(\sigma)=D \sum_{i} \sigma_{i}^{2}-\sum_{\text {neighbors }(i, j)} \sigma_{i} \sigma_{j} \text {, }
$$

where $D$ is the number of neighbors to each element and the last sum goes over all neighboring cells. This prior already favors more realistic conductivity profiles, as seen in Fig. 2(b). Still it has a tendency to result in bended surfaces, leading to different conductivity distributions as a function of height at different positions. This is expected as a horizontal shape of the conductivity is not used in the construction of the prior. Therefore we develop a prior distribution, which is taylored towards the expected result in our case.

\section{Derivation of a Height Prior}

We have seen that the priors often used are not very well suited in order to reconstruct the conductivity pattern for the phase boundary found in our case. We therefore derive here a "height-prior", which is able to capture this.

Following [8] the regularization matrix is given by the inverse of the correlation between the conductivities

$$
\left(M^{-1}\right)_{i j}=C_{i j}=C\left(\sigma_{i}, \sigma_{j}\right)
$$


where $C$ is the matrix describing the correlation between the conductivities from the uncorrelated distributions. The uncorrelated distributions in our case are the conductivity patterns for different heights $h$. In terms of the height $h$ the conductivity is given by

$$
\sigma(x, h)=\sigma_{0} \Theta\left(h-h_{i}\right)
$$

with $\Theta$ the Heaviside function and $h_{i}$ the height corresponding to $x_{i}$.

From this we can calculate the correlation

$$
C_{i j}=\int_{0}^{H} \frac{d h}{H} \sigma\left(x_{i}, h\right) \sigma\left(x_{j}, h\right),
$$

where the have assumed that the distribution of the different heights $h$ is uniform. This integral can be solved:

$$
C_{i j}=\frac{\sigma_{0}^{2}}{H} \min \left(h_{i}, h_{j}\right) .
$$

If we now reorder the positions $x_{i}, x_{j}$ in our model, such that they are increasing as a function of the new indices i' and j'

$$
h_{i^{\prime}}<h_{j^{\prime}} \text { for } i^{\prime}<j^{\prime}
$$

and assume that the positions are distributed uniformly over the whole height. We get

$$
h_{i^{\prime}} \approx i^{\prime} \frac{H}{N}
$$

With $N$ the number of cells of our model. With this the correlation matrix is

$$
C_{i^{\prime} j^{\prime}} \sim \min \left(i^{\prime}, j^{\prime}\right)
$$

The inverse of this matrix is known $[9,10]$ and of a tridiagonal form, giving the height prior as

$$
M_{\text {height }, i^{\prime} j^{\prime}} \sim\left(\begin{array}{cccccccc}
2 & -1 & 0 & 0 & \ldots & 0 & 0 & 0 \\
-1 & 2 & -1 & 0 & \ldots & 0 & 0 & 0 \\
0 & -1 & 2 & -1 & \ldots & 0 & 0 & 0 \\
\ldots & & & & \ldots & & & \ldots \\
0 & 0 & 0 & 0 & \ldots & 2 & -1 & 0 \\
0 & 0 & 0 & 0 & \ldots & -1 & 2 & -1 \\
0 & 0 & 0 & 0 & \ldots & 0 & -1 & 1
\end{array}\right)_{i^{\prime} j^{\prime}}
$$

We have tested this result also using the FEM mesh used as a basis. Calculating the prior distribution numerically it is found to be very close to the analytical result above. Therefore we have made use of this in our reconstruction.

The interpretation of the result is intuitive: The prior has a structure similar to the Laplace prior used above. But by ordering the different elements according to their heights, the prior favors similar conductivities for those elements, which are close together with respect to their height, not as neighbors in general.

The result of the reconstruction are shown in Fig. 2(c). Compared to the result of the Laplace prior, we find a conductivity pattern, which is now similar to the expected one. Therefore a good prior distribution is essential in our case to get a good reconstruction of the height. We have also compared the result for different filling heights. We have seen that it is important as well to find reasonable value of the regularization parameter $\lambda$. In our case we know, that the conductivity at the upper end of the pipe should be zero. Therefore we adjust the regularization parameter such that this boundary value is found. Results 
of the conductivity profile for different positions along the path and for two different heights are shown in Fig. 2(d). The reconstruction shows a difference between the two filling heights, but always gives a rather broad transition between the two regions. Due to the use of the height-prior the conductivity profile is more or less independent on the position along the pipe.

Up to now we have mainly used a 2D reconstruction procedure. This is already a numerically intense task, but one could ask, whether a full 3D reconstruction is needed in order to get realistic results from real measurements. We have therefore investigated 3D reconstructions as well. As we are not able to resolve any conductivity pattern along the pipe axis, we have used a 2D-to-3D mapping of the conductivity profile for this. The conductivity distribution is only determined in a plane and then mapped along the third dimension. We have found that this does not enhance significantly our reconstruction accuracy but is computationally much more demanding.

Reconstruction has been done for both simulated and real results. We have found similar results for the reconstruction in both cases. We therefore conclude that we are able to reconstruct the conductivity distribution using ERT in our system. The smoothness of the conductivity pattern makes a precise determination of the phase boundary not very accurate. The advantage of the approach is its flexibility in the reconstruction also for different patterns.

We have found also that the computing power required for the reconstruction is beyond what is available in typical flowmeter systems, even if the height needs to be determined only at a low rate. We have therefore investigated the possibility to reduce the complexity even further. One approach is to allow only for a parameterized conductivity profile. In our case this is a conductivity distribution of the form

$$
\sigma\left(x_{i}\right)=\sigma_{0} \Theta\left(h-h_{i}\right) .
$$
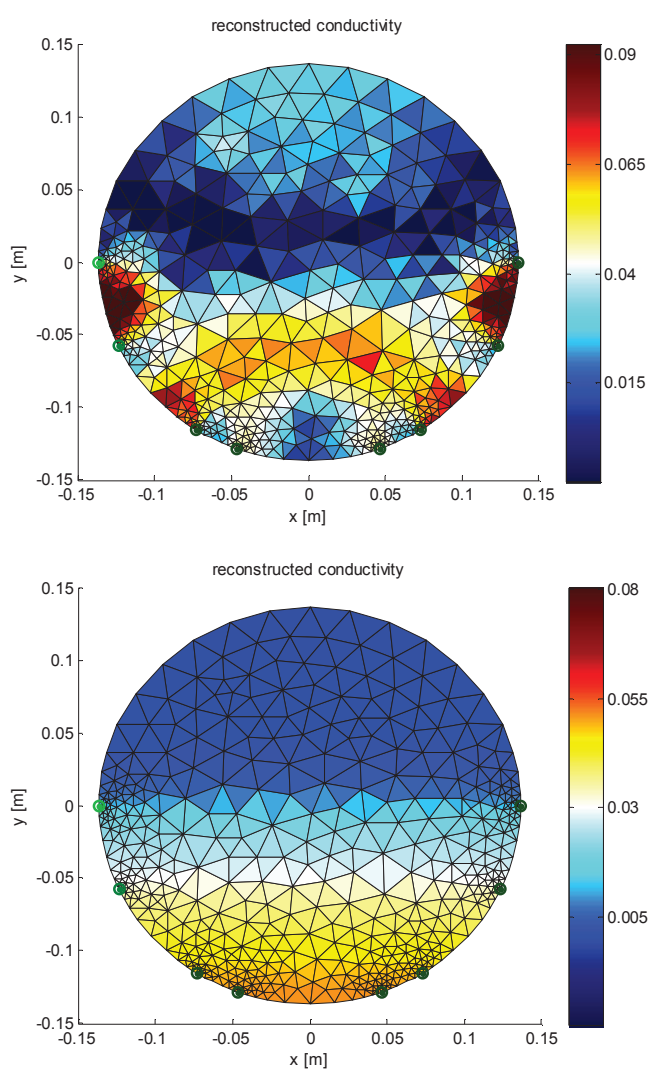

(a)

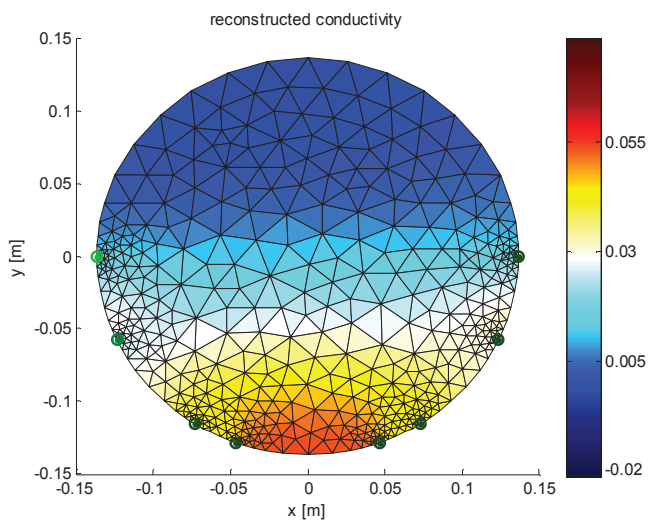

(b)

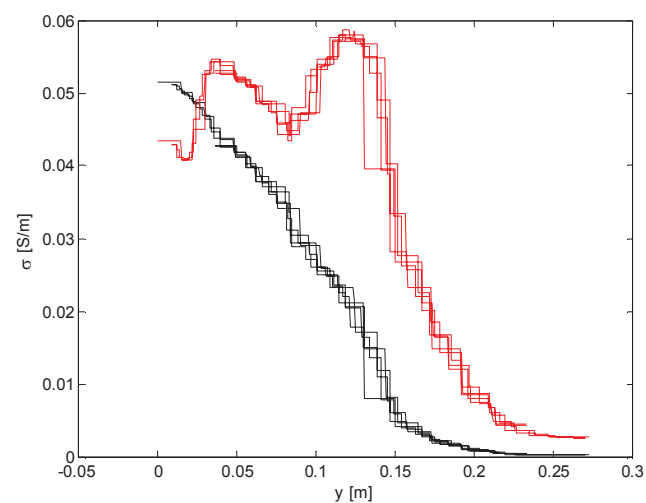

(c)

Figure 2: Reconstruction of the conductivity using different priors; (a) shows the result using a Tikhonov prior, (b) for a Laplace prior and (c) for the height prior. (d) shows the comparison of the reconstructed conductivity as a function of the height for different positions in the pipe. Results for a $55 \%$ (black) and $66 \%$ (red) filling are shown. 
This conductivity distribution only depends on the two parameter $\sigma_{0}$ and $h$. The optimization algorithm is then much faster. Also a regularization scheme is not needed anymore. We have tested this possibility as well. We have found that using this approach, the accuracy of the reconstructed height, especially at high filling heights, close to the upper end, can be improved.

\section{Summary and Conclusions}

We have investigated the possibility to use ERT methods in a magnetic flowmeter using multielectrodes in order to reconstruct the height of a partial filling. We have seen that the electrode configuration is sensitive towards the filling height, but in order to reconstruct the conductivity profile in a reasonable way, we need to provide prior information about the expected conductivity profile by using a specially designed prior. We derive a prior, which takes into account the shape of the conductivity profile in our case. We see that this allows to get more realistic conductivity distributions. This gives a good compromise for the expected profile, still allowing for a flexible profile. Different filling levels can be distinguished in this way. Still the reconstruction gives a rather broad phase boundary.

The use of EIDORS has been an advantage in our case, as the openess of the source code allows us to add the new prior in a simple way.

\section{Acknowledgement}

The authors would like to thank David Boillat, Hans Zellweger and Michael Walter, who contributed significantly to this work during different stages.

\section{References}

[1] "Theory of Electromagnetic Flow-Measurement", J. A. Shercliff, Cambridge University Press, 1962.

[2] „Der inductive Durchflussmesser mit inhomogenem Magnetfeld“, W. L. Engl, Archiv für Elektrotechnik, Vol. 53, 1970 and Vol. 54, 1972.

[3] "Electromagnetic Flowmeter FXP4000 (PARTI-MAG II), The system to meter waste water in full and partially full pipe lines", ABB 2008.

[4] "Proceedings of the 5th World Congress on Industrial Process Tomography", $3^{\text {rd }}-6^{\text {th }}$ September 2007, Bergen.

[5] "Image Reconstruction Algorithms for Soft-Field Tomography", Nick Polydorides, Ph.D. Thesis U Manchester 2002.

[6] "Uses and abuses of EIDORS: An extensible software base for EIT", Andy Adler, William R B Lionheart, Physiol. Meas. 27:S25-S42, 2006

[7] EIDORS Homepage: http://eidors3d.sourceforge.net/

[8] "Information Theory, Inference and Learning Algorithms", David McKay, Cambridge University Press, 2003

[9] "Eigenvalues and eigenvectors of two symmetric matrices", W.F. Trench, IMAGE Bulletin of the International Linear Algebra Society 22 (1999) 28-29.

[10] "On the eigenvalues of some tridiagonal matrices", C. M. da Fonseca, Journal of Computational and Applied Mathematics 200 (2007) 283 - 286- 